\title{
Structural Characterization of ZnTe Grown by Atomic-Layer-Deposition Regime on GaAs and GaSb (100) Oriented Substrates ${ }^{1}$
}

\author{
Roberto Saúl Castillo-Ojeda ${ }^{a}$,Joel Díaz-Reyes ${ }^{b *}$, Miguel Galván-Arellano ${ }^{c}$, Francisco de Anda-Salazar ${ }^{d}$, \\ Jorge Indalecio Contreras-Rascon ${ }^{e}$, María de la Cruz Peralta-Clara ${ }^{b}$,Julieta Salomé Veloz-Rendón ${ }^{b}$ \\ ${ }^{a}$ Universidad Politécnica de Pachuca, Carretera Pachuca-Cd. Sahagún Km. 20, Rancho Luna, \\ Ex-Hacienda de Sta. Bárbara, Zempoala, Hidalgo, 43830, México \\ ${ }^{b}$ Centro de Investigación en Biotecnología Aplicada, Instituto Politécnico Nacional, Ex-Hacienda de \\ San Molino, Km 1.5 de la Carretera Estatal Santa Inés Tecuexcomac-Tepetitla, Tepetitla de Lardizábal, \\ Tlaxcala, 90700, México \\ cDepartamento de Ingeniería Eléctrica, Sección de Electrónica del Estado Sólido, Centro de Investigación \\ y de Estudios Avanzados, Instituto Politécnico Nacional, Apartado Postal 14-740, City of México, \\ 07000, México \\ 'Instituto de Investigación en Comunicación Óptica, Universidad Autónoma de San Luis Potosí, Ave. \\ Karakorum 1470, Lomas $4^{a}$ Sección. Álvaro Obregón, 64, San Luis Potosí, 78210, México \\ ${ }^{e}$ Departamento de Física, Universidad de Sonora, Apdo. Postal 1626, Hermosillo, Sonora, 83000, México
}

Received: February 29, 2016; Revised: October 21, 2016; Accepted: November 21, 2016

This work presents the characterization of ZnTe nanolayers grown on GaAs and GaSb (100) substrates by the Atomic Layer Deposition (ALD) regime. Under certain conditions, the alternating exposition of a substrate surface to the element vapours makes possible the growth of atomic layers in a reactor where the atmosphere is high-purity hydrogen. ZnTe was grown simultaneously on GaAs and $\mathrm{GaSb}$ at the same run, allowing, a comparison between the effects produced by the superficial processes due to the different used substrates, thereby eliminating possible unintended changes of growth parameters. Nanolayers on GaSb maintained their shiny appearance even at temperatures near $420^{\circ} \mathrm{C}$. It was found that for exposure times below $2.5 \mathrm{~s}$ there was not growth on $\mathrm{GaAs}$, while for $\mathrm{GaSb}$ the shortest time was $1.5 \mathrm{~s}$ at $385^{\circ} \mathrm{C}$. By HRXRD the peak corresponding to (004) diffraction plane of $\mathrm{ZnTe}$ was identified and investigated, the FWHM resulted very wide (600-800 arcsec) indicating a highly distorted lattice mainly due to mosaicity. Raman scattering shows the peak corresponding to LO-ZnTe, which is weak and slightly shifted in comparison with the reported for the bulk ZnTe at $210 \mathrm{~cm}^{-1}$. Additionally, the measurements suggest that the crystalline quality have a dependence with the growth temperature.

Keywords: Atomic Layer Deposition, nanolayers, X-ray diffraction, Raman scattering

\section{Introduction}

Nowadays, most of the modern electronic devices are based on the growth of nanometric layers, strained or not; superlattices, quantum wells, optical modulators, hotelectron transistors, are only a few examples of the wide field of application of them ${ }^{1-4}$. Among the most successful semiconductor materials for the radiation detection, ZnTe has excellent optical properties as its quantum efficiency. ZnTe has proved to be a very successful material for the radiation detection in its bulk form although is a promising material for radiation detection in its nanolayers form ${ }^{5,6}$. The layer by layer growth regime can be implemented by a precise control of the precursor flows in systems as CVD, MOCVD, MBE, Radical-Enhanced ALD ${ }^{7,10}$. Normally, this is done by exposing the substrate, in an alternating way to the

${ }^{1}$ Article presented and selected from "The XXIV International Materials Research Congress (IMRC) 2015". Symposium 5B "Structural and Chemical Characterization of Metals, Alloys and Compounds". Cancun, Mexico August 16-20, 2015.

* e-mail: joel_diaz_reyes@hotmail.com vapour of each one of the constituent elements of the layer, followed by a purge or dead period between exposures in order to evacuate adequately the growth chamber. An important characteristic of ALD is its self-regulated nature ${ }^{7-10}$. In this work are presented the results obtained of the growth and characterization of ZnTe epitaxial layers on GaSb and GaAs by ALD regime under an ambiance of high purity hydrogen at atmospheric pressure.

\section{Experimental Procedure}

The growth system used in the experiments is depicted in Figure 1, which has a horizontal geometry; the body of the reactor is made of a quartz tube while the suceptor is made of high purity graphite. The experiments were performed using a high purity Pd-Ag diffused hydrogen flow of $300 \mathrm{ml} / \mathrm{min}$. 


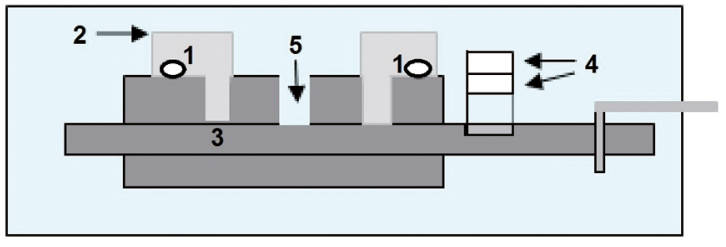

Figure 1: Details of suceptor used for growing the samples. 1) Elemental source, 2) quartz container, 3) slide strip holder, 4) GaAs and $\mathrm{GaSb}$ substrates, 5) purge hole.

The substrates are exposed alternatively to the precursor vapours by sliding a graphite rule containing them. The elemental precursor vapours arrive on the substrate surface from little quartz tubes where are contained. In this way both of them, the anion flux and the cation flux saturate the growth surface one after the other taking advantage of the self-regulated process characteristic ${ }^{11-13}$. All, the graphite suceptor, substrates and precursor sources were maintained at the same temperature. The graphite rule with the substrates is coupled to a step motor controlled by a computer program. This motor is located outside of the growth chamber. The computer program allows sequencing the layer growth and provides the desired exposure time.

The substrates preparation was as usually; degreased with organic solvents, oxide elimination using $\mathrm{HCl}$ followed by a chemical polishing. In the case of the GaAs substrates the used etching solution was $\mathrm{H}_{2} \mathrm{SO}_{4}: \mathrm{H}_{2} \mathrm{O}_{2}: \mathrm{H}_{2} \mathrm{O}, 5: 1: 1$, while in the $\mathrm{GaSb}$ case was used a solution based in tartaric acid, hydrofluoric acid and hydrogen peroxide ${ }^{11}$. After the chemical etching, the substrates were rinsed in deionized water and dried by blowing nitrogen on their surfaces. As next step, the substrates were introduced into the growth chamber and were placed side by side on a graphite rule. In order to guarantee the same growth conditions on both $\mathrm{GaSb}$ and GaAs substrates, the two substrates were used simultaneously at the same growth run. This was accomplished cutting rectangular substrates of $0.5 \mathrm{~cm} \times 1.0 \mathrm{~cm}$ and placed side by side to form a $1.0 \mathrm{~cm} \times 1.0 \mathrm{~cm}$ substrate. In this way were eliminated possible factors introduced by nonintentional variation in the growth processes assuring the same growth conditions for the deposited layers on both substrates and allowing the direct comparison between the grown layers on $\mathrm{GaSb}$ and $\mathrm{GaAs}$ substrates.

In order to study the structural characteristics of the epitaxial layers grown with different temperatures and thicknesses, high-resolution X-ray diffraction (HRXRD) measurements were performed. All the X-ray diffractograms were acquired using a Philips Analytical diffractometer. For scanning the used parameters were: $\mathrm{Cu} \mathrm{K}_{\alpha 1}$ line with wavelength of 1.5406 $\AA, \Delta \lambda / \lambda=2 \times 10^{-4}$, and $0.10 \mathrm{sec}$ as time per step, the reflection plane was (004), with a continuous mode scan type. All the used substrates were cleaved from the same epi-ready $\mathrm{GaSb}$ wafer and in the same way in the case of the GaAs substrate. For assessing the thickness of the grown $\mathrm{ZnTe}$ layers, ellipsometric measurements were performed using a Gaertner ellipsometer L117 with and He-Ne laser with $\lambda=632.8 \mathrm{~nm}$, varying the incident angles and obtaining the extinction parameters. Raman scattering experiments were performed at room temperature using the red laser at $\lambda=6328 \AA$ at normal incidence. The light was focused in a circular spot of $6 \mu \mathrm{m}$ diameter on the sample using a $50 \times$ (numerical aperture 0.9) microscope objective. The nominal laser power used in these measurements was $20 \mathrm{~mW}$. Care was taken to avoid the heating of the sample inadvertently to the point of changing its Raman spectrum. Scattered light was analysed using a Dilor micro-Raman spectrometer, with a holographic notch filter made by Kaiser Optical System, Inc. (model superNotch-Plus), a 256×1024-pixel CCD used as detector, and two interchangeable gratings (600 and 1800 $\mathrm{g} / \mathrm{mm}$ ). Typical spectrum acquisition time was limited to 60 $\mathrm{s}$ to minimize the sample heating effects discussed above. Absolute spectral feature position calibration to better than $0.5 \mathrm{~cm}^{-1}$ was performed using the observed position of $\mathrm{Si}$, which is shifted by $521.2 \mathrm{~cm}^{-1}$ from the excitation line.

\subsection{ZnTe Growth Procedure}

The experiments were carried out in a wide temperature range but $\mathrm{ZnTe}$ layers with a shiny surface could be grown only at temperatures between 370 and $400^{\circ} \mathrm{C}$ on both $\mathrm{GaSb}$ and GaAs substrates. In the case of the GaAs substrates, for temperatures above $400^{\circ} \mathrm{C}$, the layer surface deteriorated and showed a milky aspect but on GaSb the layer surfaces kept their shiny appearance even at temperatures near $420^{\circ} \mathrm{C}$. In other set of experiments, the exposure times to $\mathrm{Zn}$ and $\mathrm{Te}$ vapours were explored, in order to determine the shortest exposure times that could be used to grow the $\mathrm{ZnTe}$ layers in the ALD regime. It was found that for exposure times below $2.5 \mathrm{~s}$ there was not growth on the GaAs substrates, while in the case of $\mathrm{GaSb}$ the shortest time was found to be $1.5 \mathrm{~s}$ for $385^{\circ} \mathrm{C}$. This difference in the exposure times indicates that the associated growth kinetic is different for $\mathrm{GaSb}$ and $\mathrm{GaAs}$ substrates. In addition, these times are not determined by the transport of the reactants on the growth surfaces. For subsequent experiments $3 \mathrm{~s}$ was taken as the exposure time for samples growth, and $3 \mathrm{~s}$ for the interruption time, as other authors have reported ${ }^{14}$. The ZnTe layer thicknesses were evaluated by ellipsometric measurements. The normalized results are summarized in Figure 2, in this one, the measured layers were grown using different numbers of exposition cycles in an interval since 300 to 900 . In figure the horizontal scale corresponds to the growth temperatures used in the experiments.

The mean value of the thickness per cycle was around $0.3 \mathrm{~nm}$ that is the value corresponding to a monolayer of $\mathrm{ZnTe}$. This indicates that the growth regime is the known as Atomic Layer Deposition (ALD). As was pointed out, the growth regime of ALD allows having a very good control on 


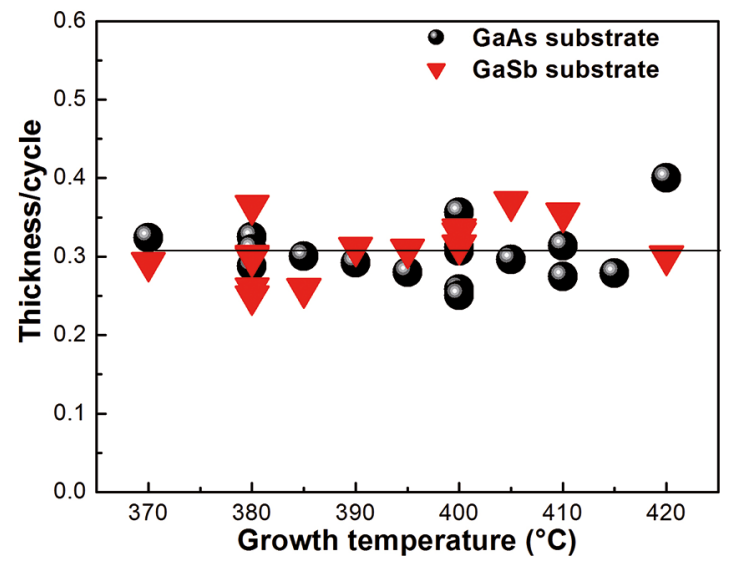

Figure 2: Thicknesses of the grown epitaxial layers as function of growth temperature.

the layer thickness, due to the close dependence of the final thickness on the total number of growth cycles performed.

\section{ZnTe X-ray Diffraction Measurements}

Figure 3 shows the X-ray diffractograms of the grown samples, as a result of the HRXRD measurements, it can be observed, the peak corresponding to the (004) diffraction plane of ZnTe layer, which was identified and investigated. The thin peak near $33^{\circ}$ corresponds to the (004) diffraction plane from the GaAs substrate and it was used as reference for the $\mathrm{ZnTe}$ layer peaks position adjustment and their comparison. The wide peaks to the left of the same one corresponding to the (004) diffraction plane from the deposited layers, their vertical intensities were amplified by 200 times due to the weaknesses of these peaks as a consequence of the thin thickness involved on the diffraction. The full width at half maximum (FWHM) of the diffraction peaks are very wide (600-800 arcsec) indicating a highly distorted lattice mainly due to mosaicity ${ }^{15}$. In addition, in this figure can be observed that the intensity of the peaks increases with the thickness of the layers. Moreover, as the layer thickness increases, the peak position moves away from the peak corresponding to GaAs substrate towards the position that must have the corresponding to the ZnTe in its bulk form. This effect can be attributed to the relaxation of the layers and the increase of dislocation defects in the interface in addition for highly dislocated layers the peak position is related with the mean distortion of the lattice and with the dislocation density generated in the relaxation process ${ }^{16}$. For the samples showed in Figure 3 the calculated thicknesses were between 135 to $270 \mathrm{~nm}$.

In the case of ZnTe layers grown on GaSb substrate was not possible to resolve clearly the ZnTe peak from the one corresponding to $\mathrm{GaSb}$ substrate. This can be attributed as consequence of the small lattice mismatch between the $\mathrm{ZnTe}$ layer and the GaSb substrate. The lattice constants of the two

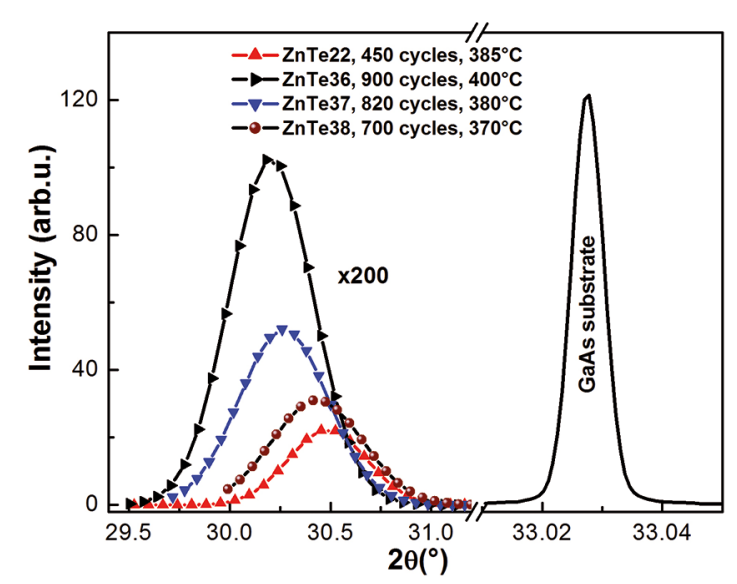

Figure 3: X-ray diffractograms of ZnTe grown on GaAs, peak position shift is related with the growth temperature and with the layer thickness.

materials are very close, $a_{\text {GaSt }}=6.0959 \AA$ and $a_{\text {ZnTe }}=6.1034$ $\AA$. In addition, the interface defects and the nanometric thicknesses of the ZnTe layers are factors related to the full width at half maximum of the diffraction peak ${ }^{17,18}$. The above described is shown in Figure 4, where is presented a $\mathrm{X}$-ray diffraction curve of a typical $125 \mathrm{~nm}$ thick ZnTe layer, obtained performing 410 growth cycles on GaSb substrate. Additionally, the peak position of the layer is closer to the peak of substrate, considering the critical thickness of 275 $\mathrm{nm}$ on (100) GaSb reported by other authors ${ }^{17}$. As a result of the deconvolution treatment of the experimental curve can be observed the peak corresponding to the $\mathrm{ZnTe}$ layer near the dominant peak corresponding to the (004) diffraction plane of the GaSb substrate. Compared with the GaSb substrate peak can be note a large peak width of the layer and its weakness is evident due to the nanometric thickness combined with the misfit dislocation density among other factors.

\section{ZnTe Characterization by Raman Spectroscopy}

Back scattering geometry has been used to record the Raman spectra of the (100) GaSb and GaAs substrates, which are shown in Figure 5. GaSb Raman spectrum which presents a dominant band that is associated to longitudinal optical phonon (LO) frequency of GaSb observed at 237 $\mathrm{cm}^{-1}$, and another which is related to metallic tellurium observed at $274 \mathrm{~cm}^{-1}$ that was found experimentally. The weak shoulder on the low-frequency side of the GaSb-LO band at $229 \mathrm{~cm}^{-1}$ is the $\mathrm{GaSb}$-TO mode, in principle forbidden in this experimental geometry. Its weak intensity in our Raman spectrum indicates that the crystalline quality of the Te-doped GaSb substrate is very good. Similarly, to GaAs substrate, which only shows a Raman peak at $293 \mathrm{~cm}^{-1}$ that is associated with LO mode. Raman scattering measurements were made to the substrates used to have a reference if there 


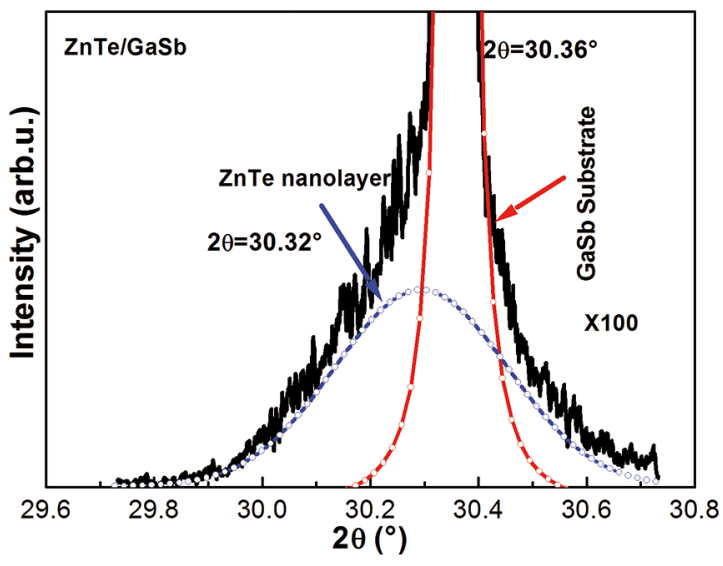

Figure 4: ZnTe and GaSb deconvoluted (004) peak. The substrate and layer peaks are difficult to resolve.

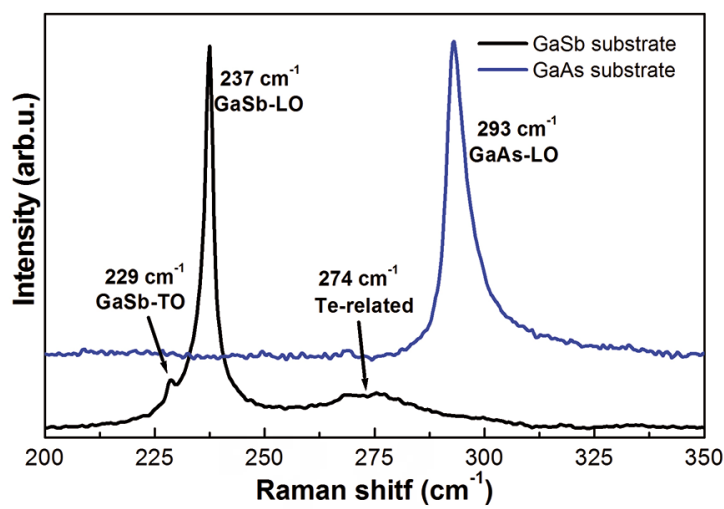

Figure 5: Raman spectra of the substrates used in the growth of thin films of $\mathrm{ZnTe}$.

had been thin film growth on different used substrates. Figure 5 shows the Raman spectra of GaAs and GaSb substrates, which have as dominant vibrational mode Longitudinal Optical (LO). And Raman spectroscopy measurements grown layers were performed for assessing the quality of the $\mathrm{ZnTe}$. The obtained results are shown in Figure 6. As was mentioned above the measurements were carried out at room temperature on samples (001) oriented with normal incidence. The layers presented in figure were grown on $\mathrm{GaSb}$ and GaAs substrates. The transversal optical (TO) mode is forbidden in the experimental measurement configuration geometry for (001) substrate orientation, it becomes active by the breakdown of the selection rules in the backscattering configuration. This breakdown is attributed to the generation of structural defects as misfit dislocation in the layer-substrate interface, inhomogeneity and other crystallinity problems originated from compositional fluctuations and by elastic scattering from ionized impurities. The peak observed at 208 to $211 \mathrm{~cm}^{-1}$ is associated to the ZnTe longitudinal optical (LO) phonon peak ${ }^{19-26}$, details of this peak are presented in

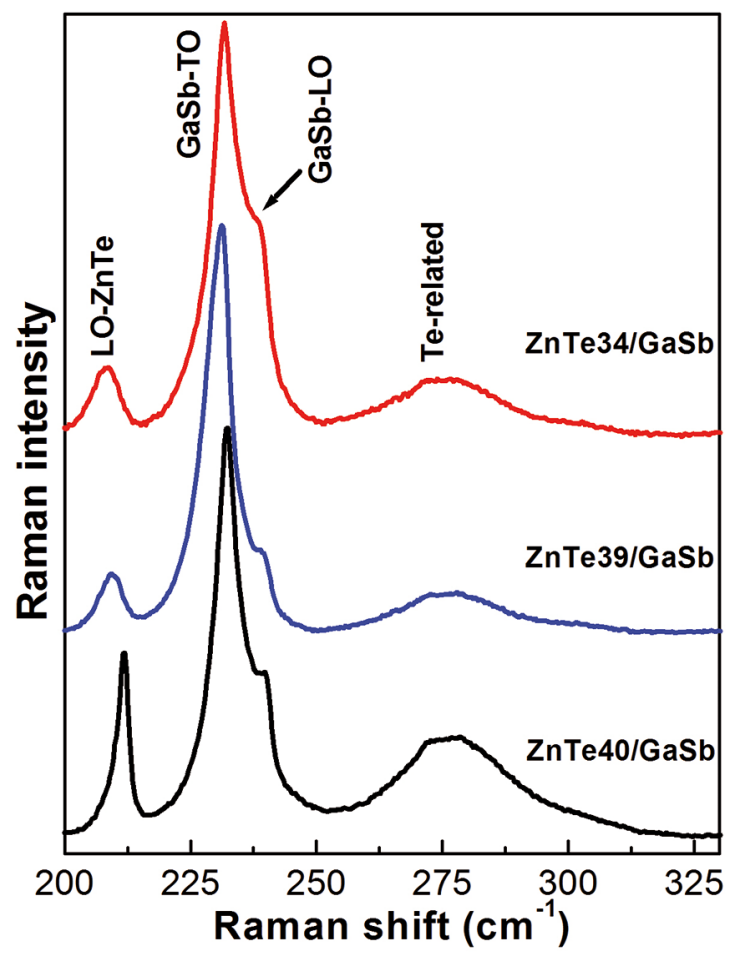

Figure 6: ZnTe Raman measurements of samples grown on GaSb. LO-ZnTe peak shows a dependence with the growth temperature as is illustrated in Table 1.

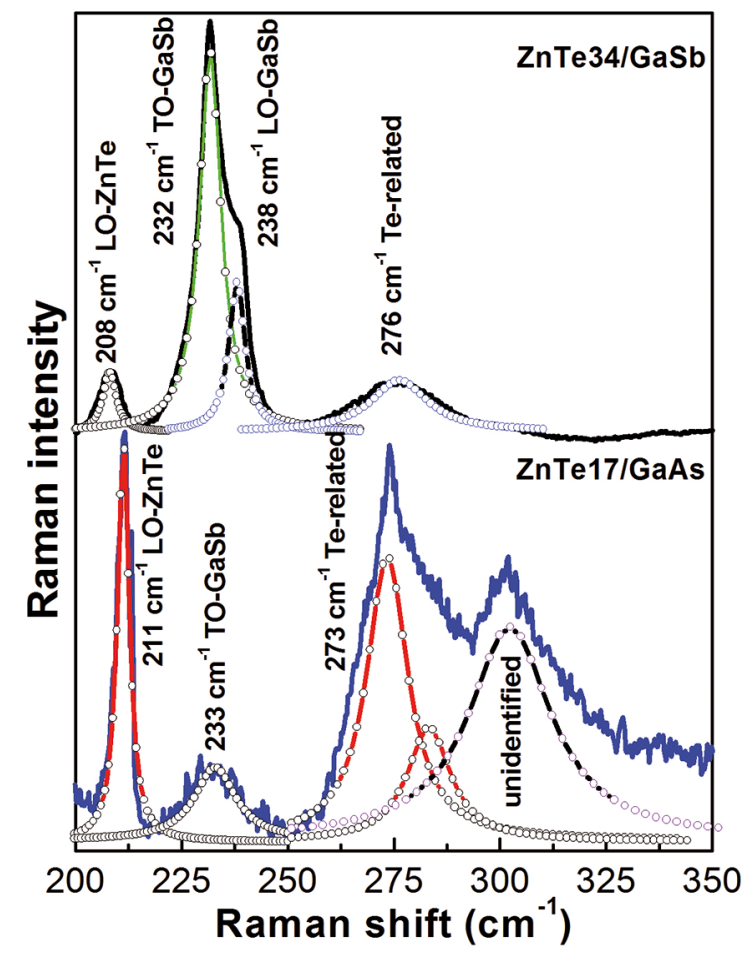

Figure 7: Raman spectra of ZnTe samples grown with different thicknesses and substrates. 
Figure 7. The peaks at 274 to $290 \mathrm{~cm}^{-1}$ are composed by the TO-GaAs and LO-GaAs vibrational modes, originated from the GaAs substrate, because of the penetration depth of laser light is larger than the $\mathrm{ZnTe}$ layer thickness and reaches the substrate, so the Raman spectra exhibit peaks related to the substrate characteristics. The peak near $231 \mathrm{~cm}^{-1}$ is the corresponding to the GaSb substrate and is composed of the TO-GaSb and LO-GaSb in $238 \mathrm{~cm}^{-1}$. In addition, the peak corresponding to LO-ZnTe shows weakness and a slightly displacement in comparison with the reported for the bulk $\mathrm{ZnTe}$ at $210 \mathrm{~cm}^{-1}{ }^{19-26}$, possibly due to the layers stress and the nanometric thickness of these layers, as has been reported by other authors ${ }^{21}$.

As can be observed in Table 1, the samples labelled as ZnTe40, ZnTe39 and ZnTe34 were grown with different temperatures, comparing the peak near $210 \mathrm{~cm}^{-1}$ the sample grown with the lower temperature shows a more intense and thin peak corresponding to the LO-ZnTe, see Figure 6. This is indicative of a dependence of the growth temperature with the crystalline quality of samples. This effect of temperature with the Raman spectra of ZnTe can be associated with the misfit dislocation increase in the interface due to the dependence of dislocation density with the temperature. The samples presented in Figure 6 were grown on GaSb with the growth temperature and thickness presented in Table 1.

As can be observed in Figures 6 and 7 the corresponding peaks coming from the substrate have the TO-GaSb peak, which must be forbidden for the experimental configuration used in the measuring due to the substrate orientation (100). In this case, it can be associated with the crystalline quality of the substrate. The appearance of this peak is indicative

Table 1: ZnTe growth conditions used for samples presented in Figure 6 grown on $\mathrm{GaSb}$

\begin{tabular}{llc}
\hline Sample & Expected thickness $(\mathrm{nm})$ & $\begin{array}{c}\text { Growth temperature } \\
\left({ }^{\circ} \mathrm{C}\right)\end{array}$ \\
\hline ZnTe34 & 165 (550 growth cycles) & 410 \\
ZnTe39 & 270 (900 growth cycles) & 400 \\
ZnTe40 & 270 (900 growth cycles) & 390 \\
\hline
\end{tabular}

of crystalline defects in the substrate, which is propagating to the layer grown of $\mathrm{ZnTe}$.

\section{Conclusions}

In this work is reported the successfully growth of $\mathrm{ZnTe}$ on both GaAs and GaSb substrates by the Atomic Layer Deposition regime as was shown from the thickness measurements performed by ellipsometry. X-ray diffraction shown a dependence of the (004) diffraction peak position with temperature and with the sample thickness which can be attributed to the lattice relaxation produced by the increase of interface defects. In addition, Raman characterization shown that the obtained samples presented a large density of crystalline defects possible dominated by misfit dislocation, additionally the samples grown at low temperature shown better Raman spectra (LO-ZnTe dominant) indicating in this way a crystalline quality dependence with the growth temperature.

\section{References}

1. Göbel EO, Ploog K. Fabrication and optical properties of semiconductor quantum wells and superlattices. Progress in Quantum Electronics.1990;14(4):289-356.

2. Ritala M, Leskelä M. Atomic layer epitaxy - a valuable tool for nanotechnology? Nanotechnology. 1999;10(1):19-24.

3. Shan CX, Fan XW, Zhang JY, Zhang ZZ, Ma JG, Lu YM, et al. Growth and characterization of ZnCdTe-ZnTe quantum wells on $\mathrm{ZnO}$ coated $\mathrm{Si}$ substrate by metalorganic chemical vapor deposition. Journal of Crystal Growth. 2001;233(4):795-798.

4. Im H, Wittenberg NJ, Lindquist NC, Oh SH. Atomic layer deposition (ALD): A versatile technique for plasmonics and nanobiotechnology. Journal of Materials Research. 2012;27(4):663-671.

5. Ruzin A, Nemirovsky Y. Methodology for evaluation of mobilitylifetime product by spectroscopy measurements in CdZnTe spectrometers. Journal of Applied Physics. 1997;82(9):41664171.

6. Nemirovsky Y, Ruzin A, Asa G, Gorelik J. Study of the charge collection efficiency of $\mathrm{CdZnTe}$ radiation detectors. Journal of Electronic Materials. 1996;25(8):1221-1231.

7. George SM. Atomic Layer Deposition: An Overview. Chemical Reviews. 2010;110(1):111-131.

8. Hsu CT. Epitaxial growth of II-VI compound semiconductors by atomic layer epitaxy. Thin Solid Films. 1998;335(1-2):284291.

9. Lee W, Kim S, Song J, Ko H, Yao T, Cho YR, et al. Molecular beam epitaxy of GaSb on ZnTe/GaAs: Influence of the chemical composition of ZnTe surface. Applied Surface Science. 2008;254(23):7728-7732.

10. Ahonen M, Pessa M, Suntola T. A study of ZnTe films grown on glass substrates using an atomic layer evaporation method. Thin Solid Films. 1980;65(3):301-307.

11. Berishev IE, de Anda F, Mishournyi VA, Olvera J, Ilyinskaya $\mathrm{ND}$, Vasilyev VI. $\mathrm{H}_{2} \mathrm{O}_{2}: \mathrm{HF}: \mathrm{C}_{4} \mathrm{O}_{6} \mathrm{H}_{6}$ (Tartaric acid): $\mathrm{H}_{2} \mathrm{O}$ Etching System for Chemical Polishing of GaSb. Journal of the Electrochemical Society. 1995;142(10):L189-L191.

12. Hartmann JM, Charleux M, Mariette H, Rouvière JL. Atomic layer epitaxy of CdTe, MgTe, and MnTe; growth of CdTe/ MnTe tilted superlattices on vicinal surfaces. Applied Surface Science. 1997;112:142-147.

13. Leskelä M, Ritala M. Atomic layer deposition (ALD): from precursors to thin film structures. Thin Solid Films. 2002; 409(1):138-146. 
14. Larramendi EM, Purón E, Hernández LC, Sánchez M, De Roux S, de Melo O, et al. Atomic layer epitaxy of ZnTe by isothermal closed space sublimation. Journal of Crystal Growth. 2001;223(4):447-449.

15. Kim BJ, Wang JF, Ishikawa $Y$, Park YG, Sindo D, Abe S, et al. Strain in the HWE-grown ZnTe/(100) GaAs hetero-interface. Journal of Crystal Growth. 2002;235(1-4):201-206.

16. Kaganer VM, Köhler R, Schmidbauer M, Opitz R, Jenichen B. $\mathrm{X}$-ray diffraction peaks due to misfit dislocations in heteroepitaxial structures. Physical Review B. 1997;55(3):1793-1810.

17. Chai J, Noriega OC, Dedigama A, Kim JJ, Savage AA, Doyle $\mathrm{K}$, et al. Determination of Critical Thickness for Epitaxial ZnTe Layers Grown by Molecular Beam Epitaxy on (211)B and (100) GaSb Substrates. Journal of Electronic Materials. 2013;42(11):3090-3096.

18. Birkholz M. Thin Film Analysis by X-Ray Scattering. Weinheim: Wiley; 2005. 378 pages.

19. Levy M, Amir N, Khanin E, Nemirovsky Y, Beserman R. Characterization of CdTe substrates and MOCVD Cd $\mathrm{Zn}_{\mathrm{x}} \mathrm{Te}$ epilayers. Journal of Crystal Growth. 1999;197(3):626-629.

20. Zielony E, Placzek-Popko E, Henrykowki A, Gumienny Z, Kamyczek P, Jacak J, et al. Laser irradiation effects on the $\mathrm{CdTe} / \mathrm{ZnTe}$ quantum dot structure studied by Raman and AFM spectroscopy. Journal of Applied Physics. 2012;112(6):063520.
21. Vinogradov VS, Karczewski G, Kucherenko IV, Mel'Nik NN, Fernandez P. Raman spectra of structures with CdTe-, ZnTe-, and CdSe-based quantum dots and their relation to the fabrication technology. Physics of the Solid State. 2008;50(1):164-167.

22. Olego DJ, Raccah PM, Faurie JP. Compositional dependence of the Raman frequencies and line shapes of $\mathrm{Cd}_{1-\mathrm{x}} \mathrm{Zn}_{\mathrm{x}}$ Te determined with films grown by molecular-beam epitaxy. Physical Review B. 1986;33(6):3819-3822.

23. Su YK, Gan KJ, Hwang JS, Tyan SL. Raman spectra of Siimplanted GaSb. Journal of Applied Physics. 1990;68(11):55845587.

24. Milekhin A, Werninghaus T, Zahn DRT, Yanovskii Y, Preobrazhenskii V, Semyagin B, et al. Raman and infrared spectroscopical investigation of the optical vibrational modes in $\mathrm{GaSb} / \mathrm{AlSb}$ superlattices. The European Physical Journal B - Condensed Matter and Complex Systems. 1998;6(3):295299.

25. Chen XK, Wiersma R, Wang CX, Pitts OJ, Dale C, Bolognesi $\mathrm{CR}$, et al. Local vibrational modes of carbon in GaSb and GaAsSb. Applied Physics Letters. 2002;80(11):1942-1944.

26. Ersching K, Campos CEM, de Lima JC, Grandi TA, Souza SM, da Silva DL, et al. X-ray diffraction, Raman, and photoacoustic studies of ZnTe nanocrystals. Journal of Applied Physics. 2009;105(12):123532. 